\title{
BYOE: A Low-cost Material Testing Machine to Increase Engagement in a Materials Science Lab Course
}

\section{Dr. Jacob Bishop, University of Nebraska, Kearney}

Jacob Bishop holds B.S. and M.S. degrees in Mechanical Engineering. He completed his Ph.D. in Engineering Education at Utah State University. His research interests are multidisciplinary. In educational research, his interests include model-eliciting activities, open online education, educational data mining, and the flipped classroom. In quantitative methodology and psychometrics, his interests focus on the use of latent variable models to analyze variability and change over time. 


\title{
BYOE: A Low-cost Material Testing Machine to Increase Engagement in a Materials Science Lab Course
}

\begin{abstract}
As a field, engineering is a profession with rich and deep theoretical foundations in each of its numerous subject areas. Helping students understand these foundational theoretical concepts can sometimes be difficult, and it is not uncommon for students to "get lost" in the details and fail to understand the main concepts. One way to help overcome this problem is to use laboratory classes. Laboratory classes provide students with hands-on learning experiences that help them connect theory and practice. One way students do this is by running experiments, collecting data, analyzing it, and comparing the results to those predicted by theoretical models. This discovery process can help build students' confidence in existing theories and help them understand these theories at a much deeper level. Although this sounds great, the reality for students in many engineering programs is different. Laboratory equipment is expensive, and even in relatively small laboratory classes (such as one with a dozen students), equipment can be overbooked. Although one or two students may get to run the equipment themselves, the rest may not get those experiences and don't benefit in the same way as the students who did.

This is the exact situation the author faced with a materials science lab course. The bottleneck in this case was the tensile testing machine, and feedback from students about the class included the fact that much of their lab time was spent "sitting around" while they waited to be able to use the (one and only) tensile testing machine. To address this problem, we developed a small low-cost tensile testing machine so that students could eventually work concurrently in groups of 2 or 3 , each with their own tensile testing machine. The current tensile testing machine prototype has a crosshead with 18 in of vertical travel and replaceable load cells of $5 \mathrm{~kg}(11 \mathrm{lb})$ to $500 \mathrm{~kg}(1100 \mathrm{lb})$. This prototype uses a dual leadscrew with a hand crank, an optical encoder to measure distance, a load cell to measure force, and a small 7" monitor to display the results in real time to the user. An Arduino board is used for data acquisition from the encoder and load cell, and this is connected to a Raspberry Pi computer, which is in turn connected to the monitor. A wireless keyboard with an integrated track pad was used to interface with the machine, whose output is shown on the small 7" monitor.
\end{abstract}

\section{Pedagogical Context}

The field of materials science is focused on connecting the concepts of structure, processing, and properties of materials. Materials science textbooks ${ }^{[4]}$ often begin with the topic of structure, then move on to properties and processing. Many students have difficulty seeing the importance of studying structure, even though the structure of materials fundamentally drives the macroscopic properties we observe, and determines the utility and potential applications. One solution to this problem is to begin with an exploration of material testing and properties, then to ask the question, "why?" A specific example is, "why does steel elongate about $25 \%$ before breaking whereas polyethylene stretches to over $500 \%$ of its original length?"

The effort to lead with properties can only be successful with functional testing hardware. Past 
efforts to do this have been met with frustration because students spend most of the time waiting for the (one and only) testing machine to become available, or merely watch others conduct tests. Another approach is to forgo physical testing altogether, and to watch videos of testing, or to perform testing simulations ${ }^{[10]}$. While there may be some benefit in watching others perform tests, it does not have the same impact as personal hands-on experience. Simulations are limited by the pre-programmed material options, and don't allow exploration beyond these limits. Universal testing machines from leading manufacturers such as Instron and Tinius Olsen cost tens of thousands of dollars each, so purchasing additional machines is not an option for most universities. The primary motivation for this work was to develop a testing platform that would enable more students to engage in hands-on learning of materials science concepts. As a tool for active learning, this builds on decades of literature on active ${ }^{[6,8]}$ and experiential ${ }^{[5]}$ learning. These both draw on the concept of constructivism ${ }^{[3]}$, which in turn relies on discovery learning ${ }^{[1,2]}$, cognitive conflict ${ }^{[7]}$, and learner-centered teaching ${ }^{[9]}$. Specific educational outcomes for a materials science course that will be served directly by this effort include:

1. Determine mechanical properties from an engineering stress-strain diagram

2. Understand concepts of stress, strain, Hooke's law, and Poisson's ratio

3. Understand and calculate true stress and strain

4. Define flexural strength and the influence of porosity for ceramics

5. Understand stress-strain behavior in polymers

6. Define hardness testing techniques and computation methods

7. Understand concepts of property variability and design/safety factors

8. Describe mechanisms of brittle and ductile fracture

9. Define creep and conditions under which it occurs and calculate steady-state creep rate

\section{Mechanical System Design and Construction}

\subsection{Testing Machine Structure}

The structure for the material testing machine was built exclusively with materials available from a local steel supplier. Plain carbon steel with a material thickness as close as possible to $1 / 8$ in was used, which allowed for high strength and easy weldability. This meant choosing either a $1 / 8$ in $(0.125 \mathrm{in})$ or $11 \mathrm{ga}(0.120 \mathrm{in})$ thickness. The list of materials used to build the steel structure and cost is shown in Table 1. Prices shown reflect those advertised by online supplier metalsdepot.com. This was done to provide an upper bound on price for anyone interested in building a similar prototype, but all the materials were actually purchased from a local supplier who had them on hand at less than half the cost listed (about \$50 USD as opposed to \$100 USD). Specific structural dimensions are shown in the CAD drawings given in Appendix A. 
Table 1: Steel Structure Materials List

\begin{tabular}{lccccc}
\hline Profile & Dimensions & Thickness & Total Length & Purchase Length & Cost* \\
\hline square tubing & $1 " x 1 "$ & $11 \mathrm{GA}$ & $86 "$ & $8 \mathrm{ft}$ & $\$ 25.16$ \\
rectangular tubing & 1 "x2" & $11 \mathrm{GA}$ & $22 "$ & $2 \mathrm{ft}$ & $\$ 12.08$ \\
channel iron & $1-1 / 2 " x 3 / 4 "$ & $1 / 8 "$ & $49.5 "$ & $6 \mathrm{ft}$ & $\$ 28.36$ \\
plate/strap & $12 "$ & $1 / 8 "$ & $16 "$ & $2 \mathrm{ft}$ & $\$ 29.50$ \\
flat bar & $3 "$ & $1 / 8 "$ & $21 "$ & $2 \mathrm{ft}$ & $\$ 6.20$ \\
flat bar & $3 / 4 "$ & $1 / 4 "$ & $8 "$ & $2 \mathrm{ft}$ & $\$ 3.88$ \\
\hline \hline
\end{tabular}

$\$ 105.18$

*Cost estimated from www.metalsdepot.com. Actual parts were sourced from a local steel supplier at a lower cost.

The base consists of a $12 \times 16$ in plate, supported on the two ends by $11 / 2 \times 3 / 4 \times 12$ in channel iron. Four lengths of 1 in square tubing are welded in a vertical position on top of the base of the structure, each set of two tubes was placed 1 in apart and the crosshead fits between them. The crosshead is made from $1 \times 2 \times 11$ in rectangular tubing oriented in the strong direction. The top of the structure is capped by a crossbeam comprised of a $3 \times 11$ in flat bar welded to a piece of channel iron with the open side facing downward. The top crossbeam is secured with four $1 / 4$ in bolts to allow for easy removal of the leadscrews. Under the base plate, another piece of channel iron 141/2 in long is welded to provide additional support and prevent the base plate from bending. This channel iron is welded with the open side facing upward, against the base plate. Further support is provided on the top of the base plate with an additional $1 \times 2$ in section of rectangular tubing welded between the vertical supports in the strong direction. Tools used for this process were an abrasive chop saw for cutting the steel to length, a grinder to remove burs, and a gas metal arc welder for joining the steel.

\subsection{Hardware}

The mechanical hardware (nuts, bolts, bushings, chain, sprockets, etc.) used for the material testing machine is listed in Table 2, and cost nearly \$100 USD. The main fabrication work for this stage of the build was in the preparation of the structure for the two lead screws, and the preparation of the lead screws themselves. The lead screws were made of common $1 / 2$ in threaded rod and the crosshead was made of $1 \times 2$ in rectangular tubing. First, an oversized (about $5 / 8$ in) hole was drilled in each end of the crosshead and lower $1 \times 2$ in structural members to allow the lead screw to pass through. Then, $1 / 2$ in coupling nuts were fitted into the ends of the rectangular tubing, so that when the lead screws are in place they support the crosshead. In this step, the sides of the crosshead tubing was bent in near the ends to hold the coupling nuts in place. Then, $3 / 8$ holes were drilled through the lower structure (base plate and channel iron), and also the upper crossmember. The two lead screws were cut to length, and one end of each was machined on a lathe to a diameter of $3 / 8 \mathrm{in}$. It is critical to have the exposed screw length closely match the distance available, so after placing the leadscrew into the lower half of the structure, the distance to the upper crossmember was measured, allowing room for the bronze bushing. The leadscrew was then removed and the other end was also machined to a diameter of $3 / 8$ in. Finally, the machine was fully assembled, and the sprockets, chain, chain guard, and handle were installed. A CAD rendering of the assembled machine is shown in Fig- 
Table 2: Test Stand Hardware

\begin{tabular}{lcc}
\hline Description & Part no.* & Total Price* \\
\hline 3/8" inside dia. 5/8 outside dia. x 1" long bronze bushing (x4) & $9440 \mathrm{~T} 55$ & $\$ 14.56$ \\
1/2"-13 x 6 ft zinc plated low carbon steel threaded rod (x1) & $98910 \mathrm{~A} 033$ & $\$ 4.89$ \\
1/2"-13 coupling nut (x2) & $90264 \mathrm{~A} 485$ & $\$ 2.04$ \\
3/8" bore finished-bore sprocket for ANSI \#25 roller chain (x2) & $2737 \mathrm{~T} 26$ & $\$ 39.62$ \\
ANSI \#25 roller chain & $6261 \mathrm{~K} 171$ & $\$ 15.42$ \\
3/8" set screw shaft collar & $6432 \mathrm{~K} 14$ & $\$ 1.07$ \\
shoulder screw, 3/8" shoulder dia. 1" shoulder length, 5/16-18 thread & $91259 \mathrm{~A} 624$ & $\$ 1.57$ \\
nylon unthreaded spacers, 3/4" OD, 1" Length, for 3/8" Screw Size & $94639 \mathrm{~A} 860$ & $\$ 0.47$ \\
polyethylene plate 5" x 8" x 1/4" & $8752 \mathrm{~K} 844$ & $\$ 7.16$ \\
1/4"-20 hex nuts (pack of 100) & $90473 \mathrm{~A} 029$ & $\$ 2.68$ \\
1/4"-13×11/2" fully threaded screws (pack of 100) & $91309 \mathrm{~A} 546$ & $\$ 8.42$ \\
\hline \hline
\end{tabular}

*Cost estimated from www.mcmaster.com. Actual parts were sourced from local suppliers at a comparable cost.

Spare bicycle chain and sprockets were used on the prototype, saving over half the listed cost.

ure 1, and the machine itself is shown in Figure 3.

\section{Electronics and Instrumentation}

Consistent with the goal of building a universal testing system that is both low in cost and easy to construct, we used an Arduino Uno R3 and a Raspberry Pi. The Arduino was chosen because of its wide availability, low power requirements, the ease with which it can be integrated with low-level sensors, and the significant community that provides countless tutorials and other support. This worked well for acquiring the raw data from the testing machine, but did not allow for easy interface, real-time data visualization, or standalone operation. In order to provide these features, a Raspberry Pi model B+ was used, together with a small seven-inch monitor. Data from the Arduino was transferred via the serial interface over a standard USB port to the Raspberry Pi. At about \$25 USD, the seven-inch monitor provided just enough viewing area, but also low power consumption. A list of the electronics used for this project is shown in Table, with the electronics for this prototype totaling approximately \$166 USD.

\subsection{Optical Rotary Encoder}

The rotary encoder used for this project had 600 pulses per revolution. With quadrature, the total resolution was 2400 increments per revolution. The encoder was connected to the interrupt pins of the Arduino (pins 2 and 3). Using interrupt routines causes the code for the position update to run when triggered by the changing state of the signal, rather than having the program constantly check the signal to determine when to update the position. This allows for accurate position tracking without bogging down the system. The encoder features a builtin voltage regulator, so it was powered externally. The input lines were protected with $10 \mathrm{k} \Omega$ pullup resistors to ensure reliability of the measurements. The wiring diagram showing the connection of both the encoder and the load cell is given in Figure. A battery is shown in the 


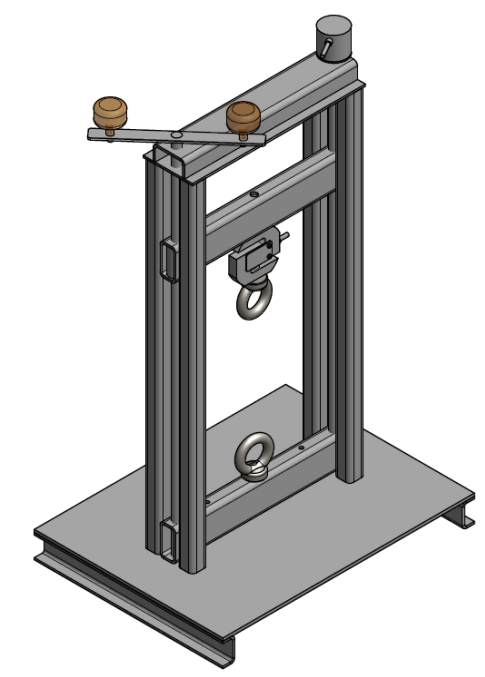

Figure 1: Isometric CAD Rendering of the Assembled Testing Machine

Table 3: Electronics and Instrumentation

\begin{tabular}{lllc}
\hline Description & Model & Vendor & Price \\
\hline Raspberry Pi 2 & B+ & amazon.com & $\$ 39.99$ \\
Arduino & Uno R3 & amazon.com & $\$ 9.99$ \\
Optical rotary encoder, 600 p/r & LPA3806-600BM & amazon.com & $\$ 17.99$ \\
$200 \mathrm{~kg}$ s-type load cell & BZLQR & amazon.com & $\$ 42.95$ \\
Fused male power socket 10A & IEC320 C14 & amazon.com & $\$ 4.47$ \\
Power cord & IEC320 C13 & amazon.com & $\$ 6.99$ \\
Proto board and wires & IB401 & amazon.com & $\$ 4.29$ \\
16 mm 5-pin aviation connector & GX12-5 & amazon.com & $\$ 8.90$ \\
24-bit A/D module & HX-711 & amazon.com & $\$ 8.11$ \\
Pyle 7 in LCD display & PLHR70 & amazon.com & $\$ 22.99$ \\
\hline \hline & & & $\$ 166.67$ \\
\hline
\end{tabular}




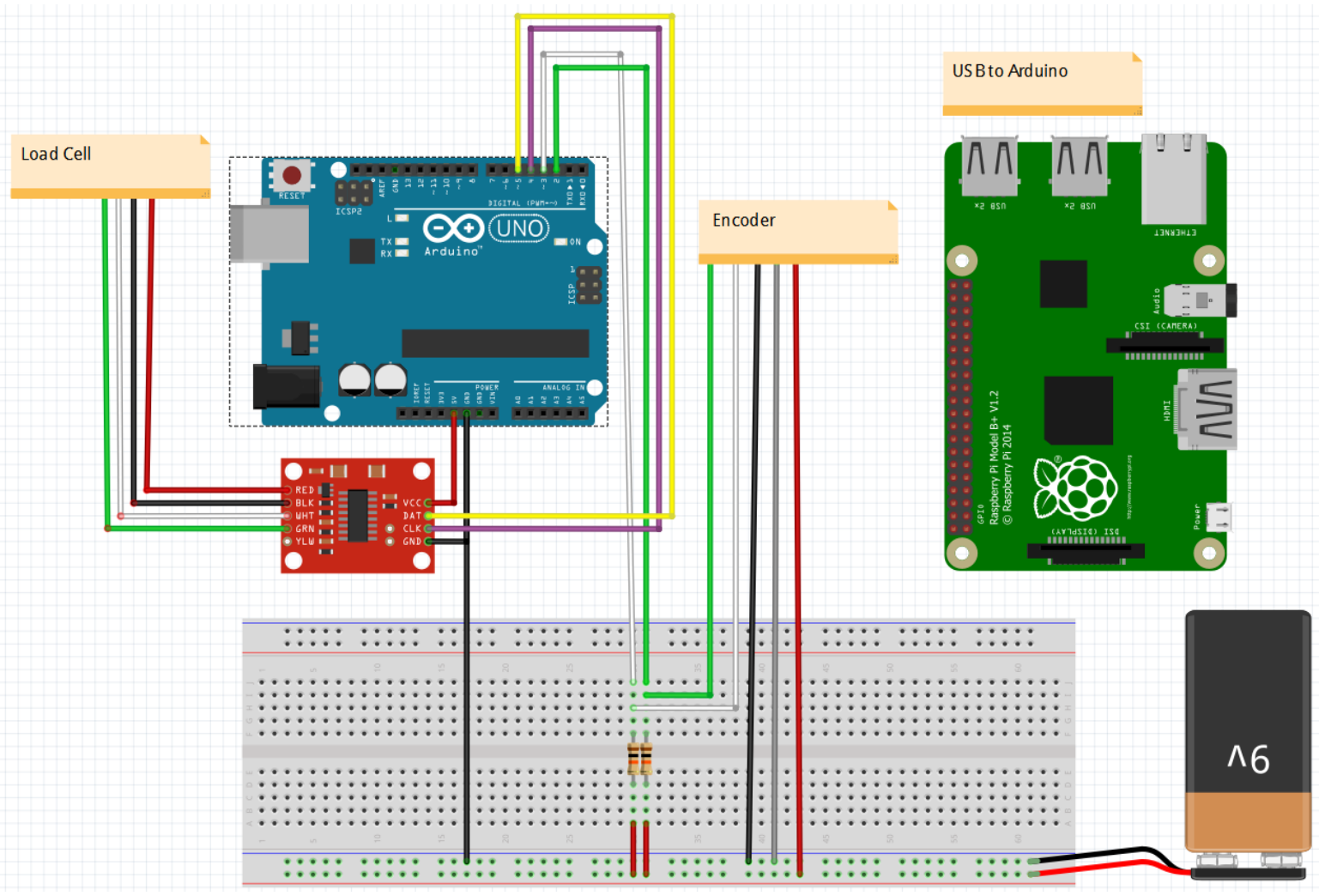

Figure 2: Fritzing diagram for the encoder and load cell 
diagram, but an $\mathrm{AC}$ adapter (wall wart) was used to provide the external 9V power for the encoder.

\subsection{Load Cell}

The Arduino has the ability to perform analog to digital (A/D) conversion, but this conversion is limited to 10 bits $\left(2^{10}=1024\right)$, yielding a number from 0 to 1023 . This is not sufficient for the purposes of measuring the input of a load cell because the voltages that need to be measured are too low. This limitation was overcome by using an external 24-bit A/D converter board, the HX711. Fortunately, there is an Arduino library available that supports the HX711 and several tutorials that were helpful in getting the load cell functioning. Depending on the application, load cells can be swapped out to optimize the measurement resolution and maximum load.

\subsection{Raspberry Pi}

The raspberry pi functions much as a standard desktop computer, and can be connected to a standard keyboard and monitor via the HDMI port. The seven-inch monitor used did not have HDMI input so an analog cable was used to connect the monitor to the raspberry pi over the composite audio/video port. A wireless keyboard with an integrated trackpad was used to interface with the testing machine.

\section{Discussion}

The completed system is shown fully assembled in Figure 3. The total cost for the materials listed was $\$ 369.75$. The cost of the system has important implications on potential applications for this project. The most obvious application for this machine is to increase the ratio of testing equipment to students to the extent that every student or pair of students in a class has the ability to work at their own machine. This is in itself a drastic improvement over the current state of affairs in which only a few students can use a machine in a class period.

Another potential use for this system is to have students build their own materials testing machine as part of the curriculum. Students could produce CAD models and part drawings as part of an introductory computer graphics course. The mechanical components could be built as part of a manufacturing course, and the electronics, instrumentation, and programming could be conducted as part of an introductory electronics and instrumentation class. Finally, the completed system could be used to conduct material tests in a materials science class. This later approach is a much more drastic recommendation than the former, but could potentially have much greater impacts as well.

\subsection{Future Improvements}

As built, the system uses standard 120 VAC power, but all of the components (including the monitor) use 12 VDC power or less, so conversion to a fully portable system with an integrated rechargeable battery power supply. A stepper motor could also be added to the system, but this would drive up the cost and reduce battery life limit the usefulness of a portable 


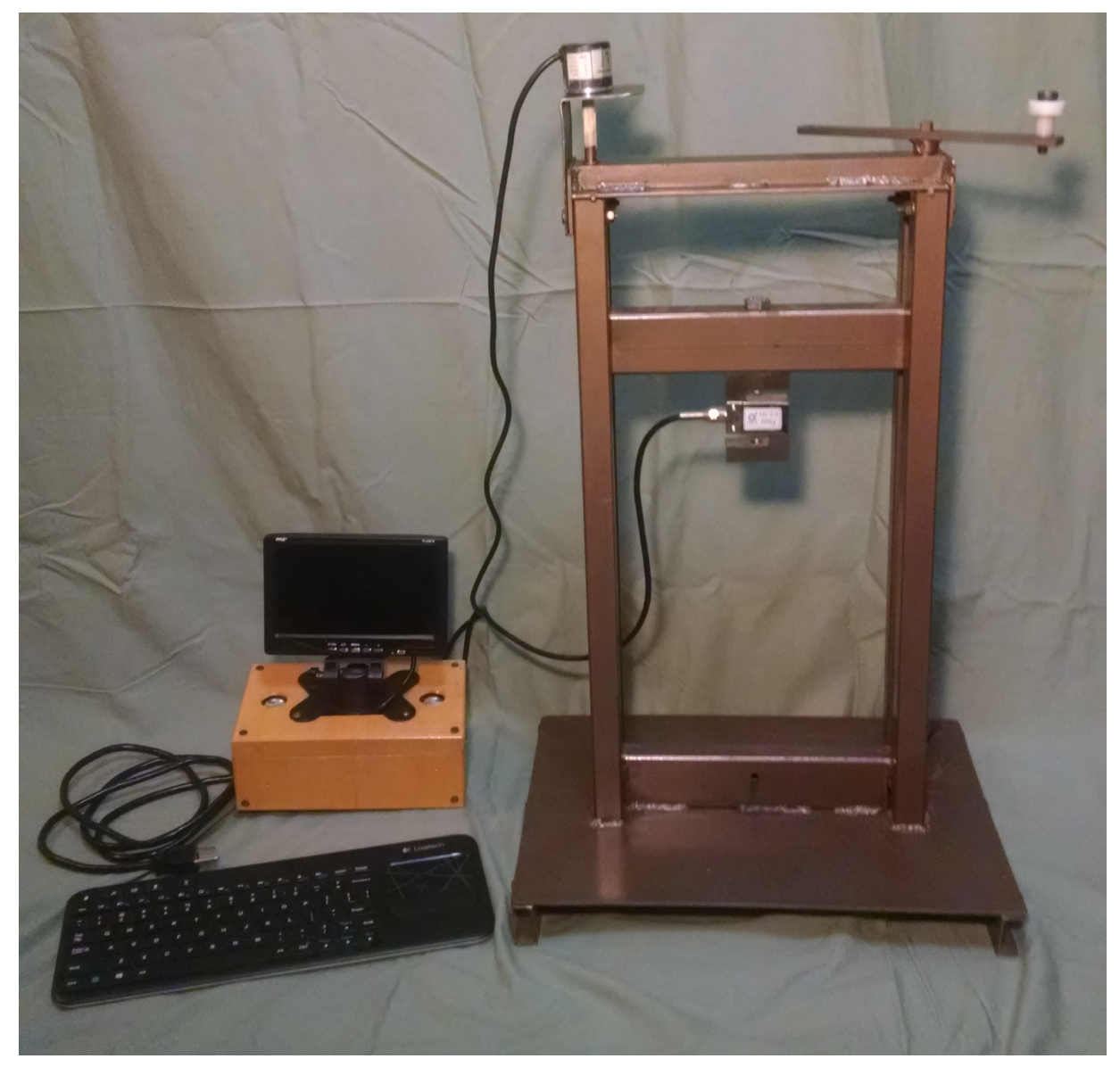

Figure 3: Assembled Material Testing Machine 
battery-powered system. Purchasing the parts and building several systems at once would reduce material waste and bring down the overall system cost.

\section{Conclusion}

A small-scale universal testing machine was designed and constructed with materials cost of less than \$400 USD. This could be used as a project for students to design and build a system as a part of three courses (Introductory Computer Graphics, Manufacturing, and Instrumentation), and use it as part of a fourth course (Materials Science).

\section{References}

[1] Jerome S Bruner. Learning and thinking. Harvard Educational Review, 29:184-192, 1959.

[2] Jerome S Bruner. The act of discovery. Harvard Educational Review, 31:21-32, 1961.

[3] Mustafa Cakir. Constructivist approaches to learning in science and their implications for science pedagogy: A literature review. International journal of environmental \& science education, 3(4):193-206, 2008.

[4] William D Callister Jr. Fundamentals of materials science and engineering. Wiley, 2001.

[5] David A. Kolb. Experiential learning: Experience as the source of learning and development, volume 1. Prentice-Hall, Englewood Cliffs, NJ, 1984. ISBN 9780132952613.

[6] J. Michael. Where's the evidence that active learning works? Advances in Physiology Education, 30(4):159167, 2006.

[7] Jean Piaget. Six psychological studies. Random House, New York, NY, 1967.

[8] M. Prince. Does active learning work? A review of the research. Journal of Engineering Education, 93:223232, 2004.

[9] C. R. Rogers. Freedom to learn. Studies of the person. Merrill, Columbus, OH, 1969. ISBN 9780675095792.

[10] Steven Wendel and Larraine A. Kapka. Virtual online tensile strength testing simulation. In Proceedings of the ASEE Annual Conference \& Exposition, New Orleans, Lousiana, June 2016. doi: 10.18260/p.27183. 
Appendix - CAD Drawing

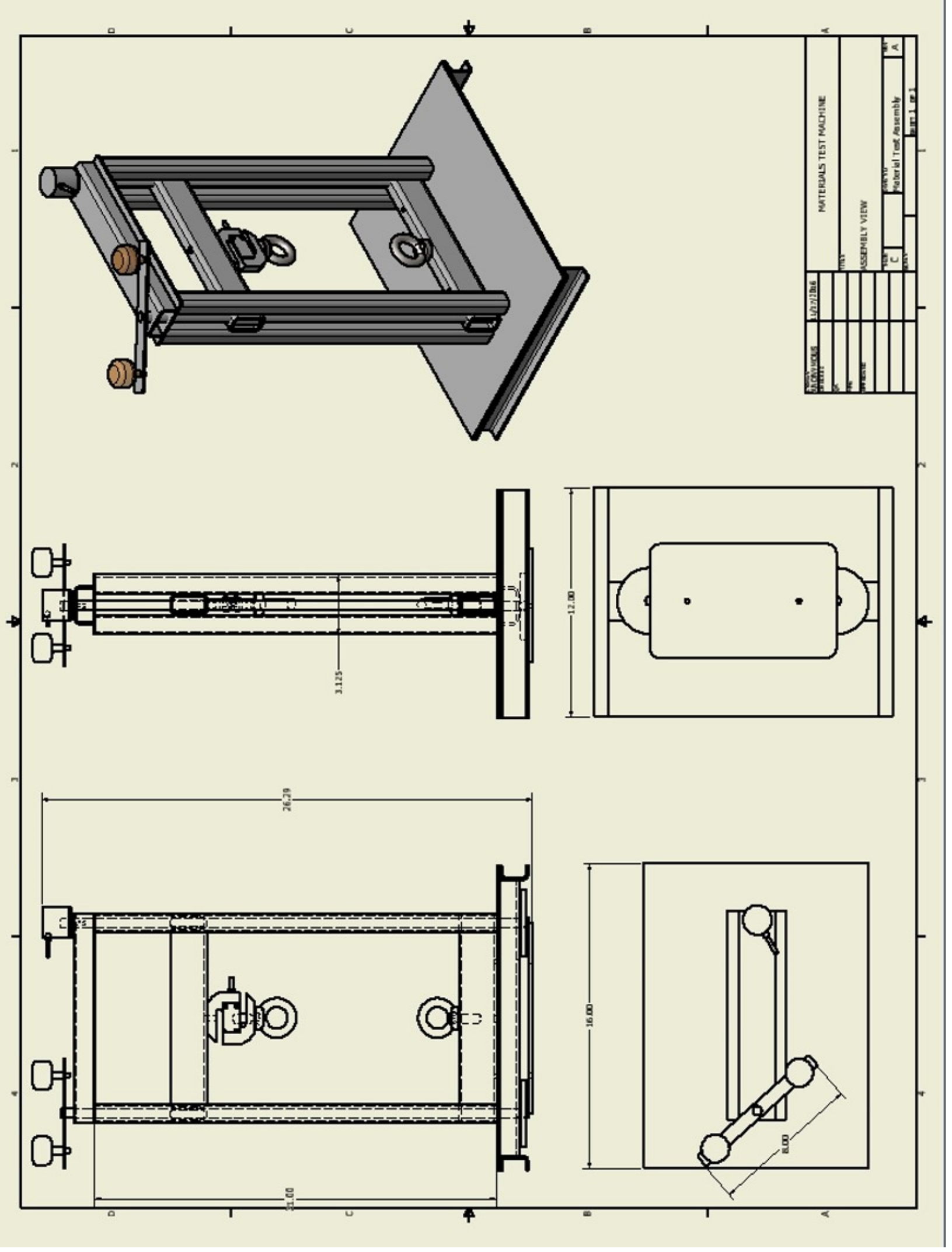

